\title{
Active control in interrupted dynamic spatial orientation: The detection of orientation change
}

\author{
JOHN F. LARISH \\ University of Illinois at Urbana-Champaign, Champaign, Illinois \\ and \\ GEORGE J. ANDERSEN \\ University of California, Riverside, California
}

\begin{abstract}
Gibson $(1966,1979)$ suggested that an important property of perception is that the observer is active. Two experiments were conducted to examine the benefits of active observation in determining dynamic spatial orientation. Subjects were presented with displays simulating locomotion through a three-dimensional environment. Active observers continuously controlled locomotion, whereas passive observers viewed the display. During the trial, the display was blacked out for a brief period, followed by a static image that was at either the correct or the incorrect orientation following the blackout. Subjects were required to indicate whether they were positioned at the correct extrapolated orientation. The presence or absence of orientation change, the type of change (changes in rotation about the depth axis [roll], horizontal axis [pitch], or forward translation), the duration of the blackout, and the consistency of change were varied. In addition, the experiments used either a compensatory or a pursuit tracking task. Active observers had greater sensitivity than did passive observers in detecting a change for both tracking tasks. Subjects in both experiments exhibited greater sensitivity in detecting inconsistent changes (relative to consistent changes), suggesting that the dynamics specified by optical flow were incorporated in extrapolated orientation. In addition, sensitivity decreased with an increase in blackout duration. The results are discussed in terms of an extrapolation model of perception that incorporates the responses executed by active observers.
\end{abstract}

An important characteristic of the ecological approach proposed by Gibson $(1966,1979)$ is that perception involves an active observer. According to Gibson, the observer is not a static organism in the environment but rather is engaged in action. Stimulation of the organism is obtained during action and not simply imposed on the organism by the environment. Gibson (1966, p. 31) further argued that action, and its effect on stimulation, can be considered as "a loop from response to stimulus to response again." This description captures the dynamic nature of control and perception that has typically been investigated in control theory (e.g., Jagacinski, 1977; McRuer, 1980; Toates, 1975).

This research was supported by an AFOSR Graduate Fellowship awarded to the first author and NSF Grant BNS 90210810 awarded to the second author. The results of Experiment 1 were presented at the 35 th annual meeting of the Human Factors Society. This study was submitted in partial fulfillment of the requirements for the $\mathrm{PhD}$ for the first author and received the distinguished dissertation award from $\mathrm{Di}$ vision 27 of the American Psychological Association. The authors would like to thank Phil Kellman and two anonymous reviewers for comments on an earlier draft. Reprint requests should be sent to either J. Larish, Department of Psychology, University of Illinois, 603 E. Daniel St., Champaign, IL 61820, or G. J. Andersen, Department of Psychology, University of California, Riverside, CA 92521.
Recently, there has been considerable interest in examining psychophysics in the context of an active observer. Warren and his colleagues (Flach \& Warren, 1993; Warren, 1988; Warren \& MacMillan, 1984) have suggested that an "active" psychophysics be considered that examines the dynamic relationship between observer and the environment during action. Interestingly, recent research in robotics has also stressed the importance of active control in three-dimensional (3-D) scene recovery by artificial intelligence systems (Brooks, 1991; Whitehead \& Ballard, 1991).

According to Gibson (1966), there are two ways of obtaining stimulation during action. One way involves exploratory or investigative action by the observer. For example, turning or orienting toward light illustrates the organisms effort to "obtain stimulation for the perception of the world" (Gibson, 1966, p. 32). The second way involves performatory or executive control, which would involve the control of performance. Steering is an example of this type of control. Both ways illustrate that stimulation is obtained by the organism, in contrast to stimulation being imposed upon the organism.

Although Gibson's arguments for the importance of active perception have been in the literature for some time, there has been relatively little research examining the benefits of active perception as compared with in- 
active or passive perception. One of the most cited studies of active control and perception is the research by Gibson (1962) involving tactile perception. Subjects were presented with a set of irregular shapes placed in the palm of the observer. In one condition, the observer's hand remained stationary while the shape was physically moved over the palm. In a second condition, the shape maintained a fixed position while the observer could physically move the palm of the hand over the shape. Thus, the first case represented passive observation, and the second case represented active observation. Gibson found that subjects were more accurate at identifying the shape if they were allowed to actively explore the shapes, providing support for the claim that active observation is important for accurate perception of the environment.

In addition, a recent series of demonstrations by Stappers (1989) provided additional support for Gibson's claim regarding the importance of active perception. Subjects were presented with computer-generated displays of 2-D shapes defined by kinetic occlusion, the deletion and accretion of texture of a far object by an opaque near object. In one condition, the rate of accretion and deletion was controlled by the observer using a computer mouse. In a second condition, the rate of accretion and deletion was constant and controlled by the computer. Accuracy in identifying the shape was greater for active control subjects, providing additional support for the importance of active observation.

The studies by Gibson (1962) and Stappers (1989) examined the first type of active perception discussed above-exploratory or investigative action. One problem with research on exploratory action is that the stimuli for active and passive observation may not be identical. In the study by Gibson, it is unclear whether the pattern of tactile stimulation was identical for passive and active observers. Likewise, in the study by Stappers, it is unclear whether the rate of accretion and deletion of visible texture was identical for passive and active observers. Because the stimuli for active and passive observation were not precisely matched, it is possible that the stimulation during active observation was more robust and, thus, resulted in improved identification performance.

The purpose of the present study was to examine the importance of the second type of active perception discussed by Gibson - performatory or executive actionby comparing the benefits of active versus passive observation in determining changes in spatial orientation. In the present study, the term spatial orientation will be used to describe the instantaneous position of the observer relative to the environment. This includes the 6 degrees of freedom (the rotation angle about the horizontal [pitch], vertical [yaw], and depth [roll] axes and position, relative to a reference point along the horizontal, vertical, and depth axes) that are commonly used to define the position and orientation of the observer with respect to the environment. Dynamic spatial orientation refers to changes in the observer's position with regard to the 6 degrees of freedom, including the direction (heading) of the observer's movement through the environment. In the present study, we examined detection of orientation changes in a subset of the 6 degrees of freedom.

Several reasons motivated us to consider the importance of active observation in determining dynamic spatial orientation. First, an important component of the definition of spatial orientation provided above is that it involves movement of the observer (locomotion). Many forms of locomotion (e.g., walking, running, swimming) involve the active control of the observer's body through the environment. In addition, other forms of locomotion (e.g., riding a bicycle, driving an automobile, flying a plane) involve the active control of a vehicle. These examples, as noted by Gibson $(1966,1979)$, can be described as closed-loop control systems in which an operator provides a response, which, in turn, results in a change in stimulation to the sense organs, which, in turn, requires another response, and so on. For example, when walking from point $A$ to point $B$ in a room, the observer takes a step (a response), which, in turn, changes the optic array, which, in turn, can lead to a new response (walk faster or slower, turn right or left to arrive at point B). Consonant with Gibson's view, we believe that the dynamic interaction between the observer and subsequent changes in stimulation in a closed-loop system captures an important aspect of active control.

Second, circumstantial evidence suggests that disorientation is influenced by whether the observer is active or passive. Reason and Brand (1975) noted that disorientation and motion sickness were more likely to occur for passive observers than for pilots flying the plane (see Armstrong, 1939, and Dhenin, 1978, for similar discussions). A study by Rolnick and Lubow (1991) found that motion sickness, which may occur during disorientation, was more likely to occur when the observer was passive (e.g., a passenger in an automobile) than when the observer was active (controlling the automobile).

Third, because our study involved computer-generated stimuli, we were able to perfectly match the visual stimulation for active and passive observers by presenting passive observers with the displays generated by the control of active observers. ${ }^{1}$

Gibson proposed that active observation was important because we, as active organisms in the environment, receive stimulation from the visual world as a consequence of our actions. Consistent with this concept, we agree with the conclusion that active observation has influenced the development of our perceptual processes and the way in which perceptual information is processed. However, unlike the arguments presented by Gibson, we propose that the benefits of active perception during performatory or executive control are the result of extrapolating future events. In terms of dynamic spatial orientation, we believe that one benefit of active perception is in estimating the orientation of the observer at some time in the immediate future. An illustration of the processes we believe to be important during active per- 
ceptual processing of a performatory nature is presented in Figure 1. The model illustrated in Figure 1 is a closedloop control system and is a variation of the sensory conflict model proposed by Oman (1982) to predict motion sickness.

According to the model, optical information is used to derive the spatial layout of the environment, speed and heading of the observer, and so on. This information is combined to form a representation of the orientation of the observer at time $T$, including both the position of the observer (the 6 degrees of freedom discussed earlier) and the rate of change of the observer's position. On the basis of the perceived position and dynamic orientation of the observer, a decision is made to respond, according to a desired goal (e.g., moving from point A to B), which is followed by a response. The executed response is combined with perceived orientation to define an extrapolated orientation of the observer at time $T+\Delta t$ (an extrapolated position and orientation in the immediate future). The response results in a change in the system (e.g., if walking, the observer takes a step forward), resulting in a change in the optic array. Perceptual processes extract the spatial layout, heading, and so on, which are used to derive a new instantaneous representation of orientation at time $T+\Delta t$. A comparison between the extrapolated and instantaneous orientation is made to determine if a new response is needed. If a new response is needed, then the observer would process information through the loop again. Processing through the loop is repeated until the desired goal is reached.

The component of the model proposed to be beneficial for active observation is the derivation of an extrapolated orientation. An active observer has two sources of information available for extrapolating orientation. One source is the execution of the response and includes information regarding the expected direction and magnitude of the change in orientation resulting from the response. The second source is the instantaneous information available from the visual scene, such as the location and orientation of the horizon and velocity information from the optic flow field. We propose that both sources are used by an active observer to extrapolate orientation. However, because a passive observer is not executing a response, extrapolating orientation can be based only on information from the visual scene. Thus, we predict that active observers will be more accurate at extrapolating orientation than will passive observers because of information available when executing the response. It is this characteristic of the model that we examined in the present study.

Our research on active and passive observation in determining spatial orientation will focus on the issue of extrapolating events-perceptual judgments following a period of no visual stimulation. This issue has been addressed by studies examining the perception of object motion. Jagacinski, Johnson, and Miller (1983) and Runeson (1975) presented displays of objects using constant velocity or constant acceleration. In Runeson's study, a linear trajectory was used; in Jagacinski et al.'s study, a parabolic trajectory was used. In both studies, the object disappeared from the display and the subject was required to report when the object would reach a specified location. Both studies found that observers predicted trajectories of the form of a constant acceleration followed by a constant velocity, rather than the actual constant velocity or constant acceleration of the object. These studies demonstrated that, although observers were able to mentally represent object motion, the representations were not veridical.

In a related series of studies, Freyd and her colleagues (Freyd, 1987; Freyd \& Finke, 1984, 1985; Freyd \& John-

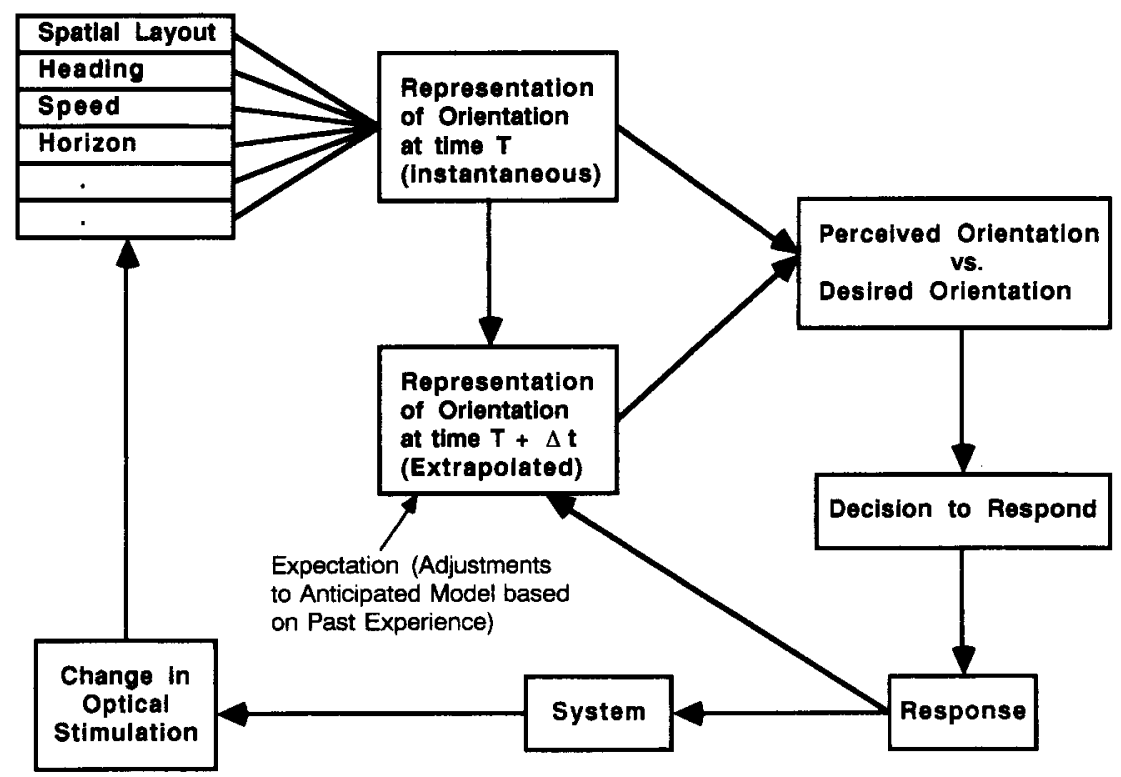

Figure 1. Schematic diagram of an extrapolating model of dynamic spatial orientation. 
son, 1987; Kelly \& Freyd, 1987) examined the importance of the dynamics of the motion of objects. Subjects were shown a sequence of three briefly presented, static rectangles in orientations corresponding to a constant rate of rotation. Following the presentation of the rectangles, a probe rectangle was presented that was at the same orientation as the third rectangle, rotated in the same direction as the implied motion, or rotated in the opposite direction as the implied motion. Subjects were required to report whether the orientation of the probe rectangle differed from the position of the third rectangle. These results indicate that the motion of the rectangles was represented with an explicit dynamic component referred to by Freyd and her colleagues as representational momentum. Representational momentum can be considered to be a direct analogue to physical momentum-the representation of the object continues to rotate on the basis of the the dynamic component of its previously perceived orientation.

While these studies suggest that human observers are able to form analogue representations of object motion, little is known about the nature of the representation used in orientation and locomotion. An important issue addressed in the present research was whether representational momentum, found in object motion, also exists for dynamic processes involving locomotion. One method of examining the representation used in extrapolating orientation is to manipulate variables that should affect the duration and decay characteristics of the representation. This method has been employed in orientation and locomotion research examining the time to contact an approaching surface (Lee, 1976; Schiff \& Detweiler, 1979). The results of these studies indicate that subjects can accurately predict the time to contact for blackout periods up to $10 \mathrm{sec}$. Similar limitations have also been found in studies involving walking and throwing tasks (Thomson, 1983).

The displays employed in the present experiments simulated observer motion through a 3-D scene. Active observers were asked to control the motion through the scene. Passive observers were asked to view the display generated by the control of active observers. At some point during the trial, the display went blank for a brief blackout period. The active observers were instructed to maintain their heading (the direction of movement through the scene) for the duration of the blackout. The blackout was immediately followed by a static image of the scene. In one condition, the static image simulated the correct orientation (pitch and roll angle) and position (along the depth axis) of the observer following the blackout. In a second condition, the static image simulated an incorrect orientation or position of the observer following the blackout. The task of the subjects was to indicate whether or not they were at the correct orientation.

\section{EXPERIMENT 1}

In Experiment 1, active subjects were required to perform a compensatory tracking task. They were instructed to maintain observer motion along the course on which each trial originated. To ensure that the task was active, the position of the observer was perturbed according to a sum of sines function, consistent with a simulation of wind gusts. These perturbations required the active observer to constantly correct the path of motion to maintain the course of travel. Passive observers were required to simply view the display.

Three variables were examined to assess the accuracy of extrapolating orientation and position. The first variable was the type of observation: passive or active observation. If the extrapolation of future events is influenced by feedback during the control of locomotion, then we expect improved performance for active observation, relative to passive observation. On the other hand, if feedback during control is not incorporated in the extrapolation of future events, then we expect no difference in detecting changes in orientation following blackout.

The second variable of importance in assessing the accuracy of extrapolating orientation and position was blackout duration. Previous research has indicated decreased accuracy in detecting changes in object motion (Freyd \& Johnson, 1987) and in locomoting to a position in the environment (Thomson, 1983) with an increase in the duration of no visual stimulation. In accordance with these results, we expect that accuracy in detecting a change in orientation will decrease with an increase in blackout duration.

Finally, a third variable examined was the consistency of change. Freyd and her colleagues (Freyd, 1987; Freyd \& Finke, 1984; Freyd \& Johnson, 1987) found that subjects' judgments of a change in object motion were more accurate when the change was inconsistent with the viewed motion than when the change was consistent with the viewed motion. On the basis of these results, Freyd and her colleagues proposed that subjects exhibit representational momentum consistent with the viewed motion. Similarly, we investigated whether a form of representational momentum is present for observer motion and orientation. Specifically, we examined whether the change in motion was consistent or inconsistent with the previously viewed motion prior to blackout. If extrapolated orientation includes the momentum present in the viewed frames prior to blackout, then increased accuracy in detecting a change should occur for inconsistent changes, relative to that for consistent changes. We examined this issue in Experiment 1 by providing changes in orientation in roll, pitch, and forward translation.

\section{Method}

Subjects. Twenty-nine paid volunteers, who were undergraduate or graduate students at the University of Illinois, participated in the experiment. The subjects had normal or corrected-to-normal vision. The data from 1 subject were not used because the subject misunderstood the instructions. The subjects were run individually and were naive with regard to the purpose of the study.

Design. Five independent variables were examined: change (present or absent), type of observation (active control or passive observation), blackout duration $(1,2$, or $4 \mathrm{sec})$, type of change (ro- 
tation about the depth axis [roll], rotation about the horizontal axis [pitch], or translation in depth), and consistency of change with prior motion (consistent or inconsistent). Type of observation was manipulated between subjects. Presence or absence of change, blackout duration, type of change, and consistency of change were varied within subjects.

Each block of trials consisted of 30 display conditions, with 12 trials in which no change occurred ( 4 at each blackout duration) and 18 trials in which a change occurred ( 3 blackout durations $\times$ 3 types of change $\times 2$ levels of consistency).

Stimuli. The displays simulated flight (forward locomotion) over simulated terrain with mountains and buildings. A rectangular surface grid and dot patterns representing city lights provided a moderate ground texture. Figure 2 depicts the type of scene used in the experiment. A crosshair superimposed over the center of the display was also present. The control dynamics simulated a highperformance twin-engine aircraft. Altitude was fixed at $61 \mathrm{~m}$ for all triais in order to maintain a consistent level of scene complexity while reducing task demands on the active controller. In addition, speed was constant at $120 \mathrm{~m} / \mathrm{sec}$. Constant control inputs were required by the active controller to maintain the desired heading. A five-wave sum-of-sines forcing function was used to simulate wind perturbations in pitch and roll. The frequencies and amplitudes for the sum-of-sines forcing function are presented in Table 1. Phases were randomized for each trial.

The stimuli consisted of a blank warning display, an active control display, a blackout display, and a static display (see Figure 3). The simulated position and orientation of the observer were determined by the control of an active observer and the forcing function described above, and they varied during both the active control and the blackout displays. The static display simulated either the correct orientation of the observer at the end of the blackout or a different (changed) orientation from the orientation at the end of the blackout. Thus, the active control, blackout, and static displays were a simplistic simulation of a pilot who flies into a cloud (blackout), must maintain control while in the cloud, and, upon exiting the cloud, must determine whether he/she is positioned at the correct extrapolated orientation.

For stimuli simulating a changed orientation, the change was a rotation about the depth axis (roll), a rotation about the horizontal

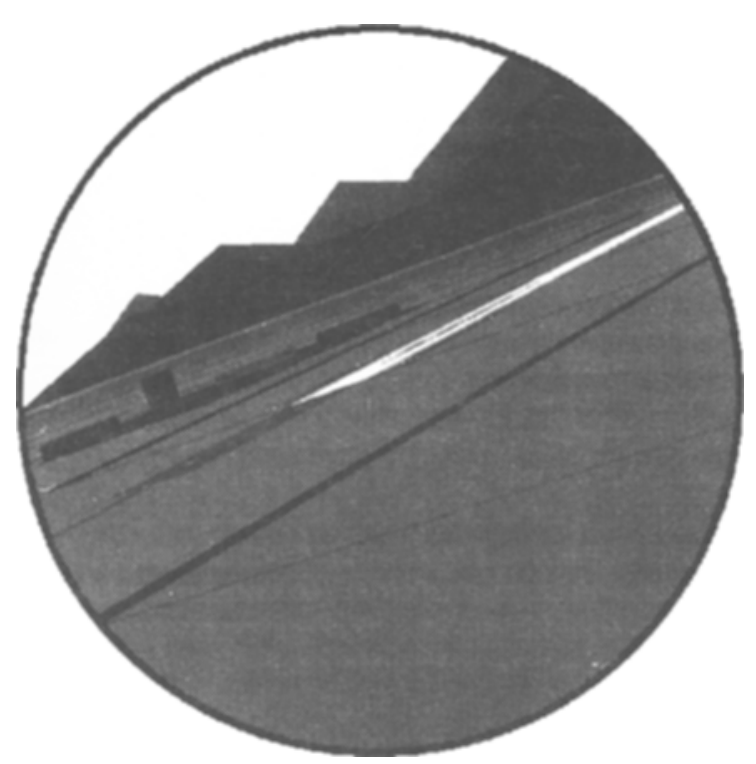

Figure 2. Representative display for Experiment 1. A crosshair superimposed over the center of the display is not depicted.
Table 1

Frequencies (in Hertz) and Amplitudes (Degrees) of Sine Waves Used in the Sum-of-Sines Forcing Functions for Experiments 1 and 2

\begin{tabular}{llll}
\hline \multicolumn{3}{c}{ Pitch } & \multicolumn{2}{c}{ Roll } \\
\cline { 1 - 2 } \cline { 4 - 4 } Frequency & Amplitude & Frequency & Amplitude \\
\hline \multicolumn{4}{c}{ Experiment I } \\
0.022 & 0.1 & 0.022 & 0.3 \\
0.05 & 0.1 & 0.05 & 0.3 \\
0.13 & 0.075 & 0.13 & 0.25 \\
0.30 & 0.015 & 0.30 & 0.05 \\
0.71 & 0.015 & 0.71 & 0.05 \\
\multicolumn{5}{c}{ Experiment 2} \\
0.347 & 0.09 & 0.347 & \\
0.593 & 0.08 & 0.547 & 0.27 \\
0.853 & 0.065 & 0.811 & 0.24 \\
1.229 & 0.045 & 1.283 & 0.195 \\
1.489 & 0.035 & 1.553 & 0.135 \\
\hline
\end{tabular}

axis (pitch), or a translation in depth. In addition, the changed orientation could be either a consistent or an inconsistent change. The consistency of changes in pitch and roll were determined by the change in orientation of the display during a 1-sec interval preceding the onset of the blackout. A consistent change in translation was a change in the direction of travel; an inconsistent change was in the opposite direction. Change consistency was determined by adding or subtracting a constant value from the correct orientation. For example, if pitch was oriented upward, a consistent pitch change was $2.5^{\circ}$ added to the correct pitch at the end of the blackout, whereas an inconsistent pitch change was $2.5^{\circ}$ subtracted from the correct pitch at the end of the blackout. The magnitude of changes in pitch and roll were determined in a pilot study and were selected to provide an approximately moderate level of difficulty. Magnitudes of change for pitch, roll, and translation were $2.5^{\circ}, 9.0^{\circ}$, and $259 \mathrm{~m}$, respectively.

Apparatus. The subject was seated in a darkened subject booth. The displays were viewed through a circular window subtending a visual angle of $25^{\circ}$. The display was collimated using a Fresnel lens $(\mathrm{fl}=0.64 \mathrm{~m}$ ) in order to reduce accommodation cues. Subject eye height was adjusted to the center of the display by raising the height of the chair, if necessary. All subject responses were made using a flightstick (Measurement Systems No. 465-G1975) attached to the right arm of the chair. Discrete responses were made using two buttons mounted in the top of the joystick. The displays were generated using a Silicon Graphics IRIS 4D70 graphics system with a high-resolution $(1,280 \times 1,024$ line $)$ color monitor. The displays generated by the control of an active observer were stored on the IRIS system and were replayed for a passive observer. Stick input was made using a personal computer connected to the Silicon Graphics system through a serial interface. The display update was $12 \mathrm{~Hz}$.

Procedure. The subjects were randomly assigned as either active controllers or passive observers. Active and passive observers were paired such that the display's controlled and viewed by an active subject were replayed to a passive subject. Prior to the experiment, the subjects were given extensive written and oral instructions on the task and required responses. Active subjects were told that they were to maintain a straight and level heading along the course on which the trial originated. They were also warned that continuous control would be necessary because of the wind buffet. The subjects in both conditions were told that a blackout would take place (as if flying into a cloud) during each trial, and they were to indicate whether they were positioned in the expected orientation following the blackout. The subjects were instructed that a change in orientation would be a change in roll, pitch, or trans- 


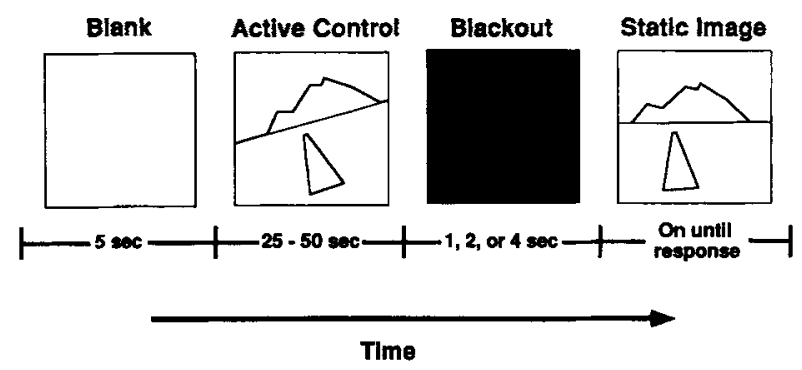

Figure 3. A schematic diagram of each trial indicating the presentation order and duration of the blank, active control, blackout, and static image displays.

lation. Active subjects were instructed to maintain the control response during the blackout that was initiated immediately prior to the blackout and not to make abrupt control responses. During the practice trials, the control movements of active observers were closely monitored. Any active observer who initiated a control response during the 30 -min practice session (prior to the experiment) was told that they were not to make control changes during the blackout. During the experiment, the control responses of the subject were also monitored by the experimenter from a second monitor in an adjacent room. The data of active subjects who initiated control changes during the blackout were not included in the study. All subjects complied with these instructions, and, thus, no data were discarded as a result of inappropriate control changes.

Each trial consisted of the following sequence of events (see Figure 3). The trial began with a blank display for 5 sec. The blank display was immediately followed by an active control display simulating motion in a straight and level orientation through the scene. The path of motion was immediately perturbed by simulated wind gusts (the sum-of-sines forcing functions). A blackout occurred following 25-50 sec of the simulated locomotion display. Following the blackout, a static scene of the simulated world was displayed. The subject was then required to indicate by pressing one of two buttons whether the scene was in the expected orientation following the blackout or whether a change had occurred. Both active and passive subjects were instructed that if a change occurred during the blackout, it would occur as a change in roll, pitch, or translation. These control changes were demonstrated with the control stick, and the subjects were allowed to familiarize themselves with the control during practice trials.

The experiment consisted of two sessions. During the first session, each subject viewed a block of 20 trials to become acquainted with the task and the response following the blackout. The subject then performed two blocks of 30 trials each. On the second day, the subject viewed two additional blocks of 30 trials each. No performance feedback was given at any time.

The 18 change conditions were presented once within each block. In addition, 12 trials in which no change occurred were presented in each block (4 at each blackout duration) for a total of 30 trials per block. Each passive subject viewed the displays previously generated by the control of an active subject. Trials were presented in a different random order in each block for pairs of active/ passive subjects. Trial order was counterbalanced across subjects.

\section{Results and Discussion}

The ability of observers to detect a change in orientation was examined. This was measured by calculating, for each observer, the hit rate (the proportion of trials in which the subject correctly identified that a change had occurred) and false-alarm rate (the proportion of trials in which a subject indicated that a change had occurred when no change had occurred) and deriving a sensitivity value $\left(d^{\prime}\right)$ for each subject in each condition. In addition, an analysis of response bias $(\beta)$ was performed for each subject for each condition. The subjects who failed to show discrimination sensitivity $\left(d^{\prime}\right.$ values greater than zero) on more than $25 \%$ of the conditions were not included in the analysis. This occurred for two sets of active/passive observers. Thus, the data analyzed in Experiment 1 were based on the responses of 24 subjects (12 active/passive pairs). Since the type of observation variable was yoked (on the basis of paired subjects who were presented with identical dynamic displays), this factor was analyzed as a repeated measures variable.

Sensitivity. A four-factor (type of observation $x$ blackout duration $X$ type of change $X$ consistency of change) analysis of variance (ANOVA) was conducted on the sensitivity $\left(d^{\prime}\right)$ of each subject to discriminate change and no-change trials. A significant main effect occurred for the type of observation $[F(1,11)=5.88$, $p<.05]$. Mean sensitivity $\left(d^{\prime}\right)$ for active and passive observers was 0.57 and 0.11 , respectively, indicating that active observers were more sensitive at detecting that a change had occurred than were passive observers.

In addition, the main effect of blackout duration was significant $[F(2,22)=3.66, p<.05]$. Mean sensitivity for the 1-, 2-, and 4-sec blackout durations were 0.62, 0.37, and 0.03 , respectively. Post hoc comparisons indicated significant differences $(p<.05)$ between the 1 - and 4-sec blackout durations. This finding suggests that sensitivity decreased with an increase in the duration of the blackout.

The main effect of type of change was also significant $[F(2,22)=41.2, p<.01]$. Mean sensitivity for changes in translation, pitch, and roll were $-0.78,1.16$, and 0.64 , respectively. Post hoc comparisons indicated significant differences $(p<.05)$ between translation and roll change and between translation and pitch change.

A significant two-way interaction was found between the consistency of change and blackout duration

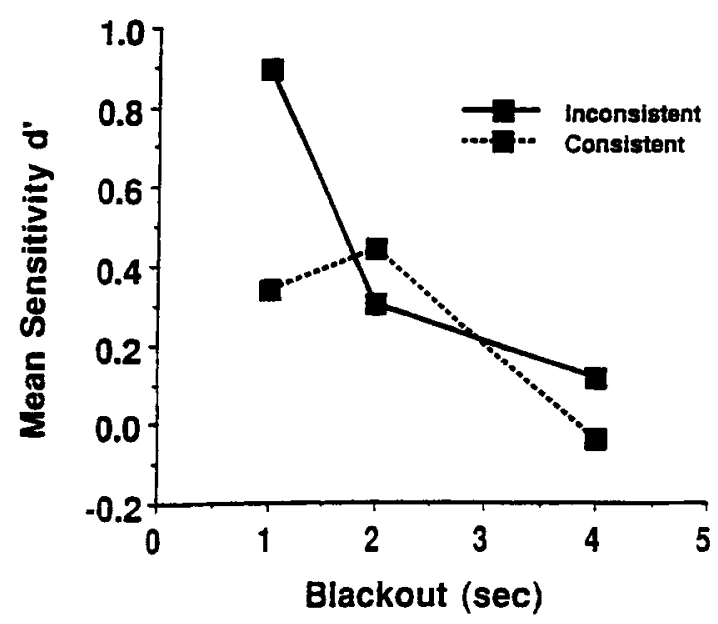

Figure 4. Effects of consistency of change and blackout duration on sensitivity in Experiment 1. 
$[F(2,22)=8.46, p<.01]$ (see Figure 4$).$ In order to examine the significant differences in this interaction, simple main effects (see Keppel, 1991, for a discussion of simple main effects) were conducted comparing differences between inconsistent and consistent change at the 1-, 2-, and 4-sec blackout durations separately. A significant simple effect was found for consistency at the 1 -sec blackout duration $[F(1,11)=15.6, p<.01]$, with greater sensitivity occurring for inconsistent change $(0.89)$ than for consistent change (0.34). The simple effects of consistency at the 2- and 4-sec blackout duration were not significant $[F(1,11)<2.0]$. Thus, the significant interaction between consistency of change and blackout duration suggests that a representational momentum effect occurred at the 1-sec blackout duration.

A significant three-way interaction was found between type of observation, consistency of change, and blackout duration $[F(2,22)=6.29, p<.01]$. As is shown in Figure 5 , the two-way interactions between type of observation and blackout duration were different for consistent and inconsistent changes. An analysis of the simple
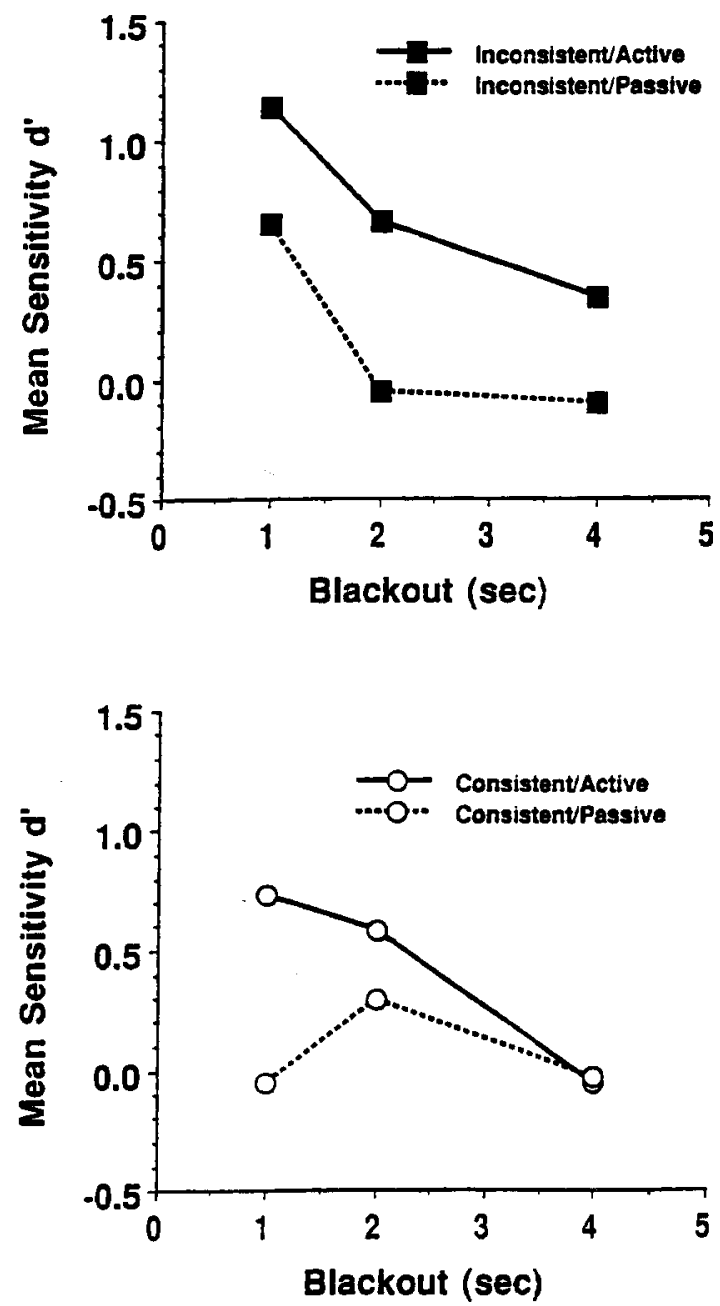

Figure 5. Effects of type of observation, change consistency, and blackout duration on sensitivity in Experiment 1.

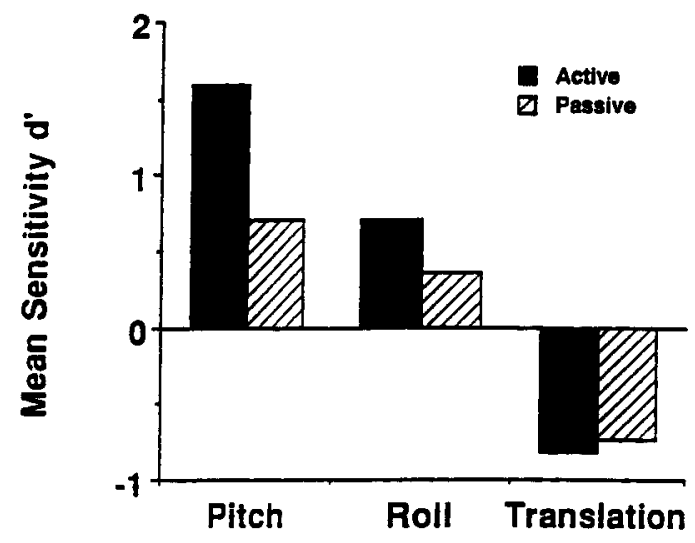

Figure 6. Effects of type of observation and type of change on sensitivity in Experiment 1 .

effects of type of observation indicated significantly greater sensitivity for active observers $(0.66)$ than for passive observers $(-0.05)$ at the 2 -sec blackout duration for inconsistent changes $[F(1,11)=10.5, p<.01]$. However, for consistent changes, greater sensitivity occurred for active observers $(0.73)$ than for passive observers $(-0.05)$ at the 1 -sec blackout duration $[F(1,11)=$ $4.79, p<.05]$. There were no other significant simple effects.

A significant two-way interaction occurred between the type of observation and the type of change $[F(2,22)=$ $4.54, p<.05]$ (see Figure 6). An analysis of the simple effects of type of observation indicated significantly greater sensitivity for active observation (1.6) than for passive observation $(0.71)$ for changes in pitch $[F(1,11)=$ $9.5, p<.05]$. No significant differences were found between active and passive observation for changes in roll and translation $(p>.05)$.

The two-way interaction between consistency of change and type of change was also significant $[F(2,22)=4.93$, $p<.05$ ] (see Figure 7). The simple effects of consistency were examined, and they revealed significantly greater sensitivity for inconsistent $(0.95)$ than for consistent $(0.34)$ changes in roll $[F(1,11)=15.5, p<.01]$. No significant differences were found for inconsistent and consistent changes in pitch and translation $(p>.05)$.

Finally, the three-way interaction of type of observation, consistency of change, and type of change was significant $[F(2,22)=4.2, p<.05]$ (see Figure 8 ). The simple effects of type of observation were analyzed for inconsistent and consistent change for translation, pitch, and roll. Sensitivity was significantly greater for active observers than for passive observers for inconsistent changes in pitch $[F(1,11)=6.4, p<.05]$, consistent changes in pitch $[F(1,11)=10.05, p<.01]$, and inconsistent changes in roll $[F(1,11)=7.5, p<.025]$. The mean sensitivity for active and passive observers was 1.5 and 0.82 for inconsistent changes in pitch, 1.71 and 0.61 for consistent changes in pitch, and 1.35 and 0.56 for inconsistent changes in roll, respectively. There were no other significant simple effects. 
An analysis was also performed to determine whether the sensitivity of active and passive observers was significantly greater than zero (Marascuillo, 1970) for the three different types of change. For active observers, the numbers of subjects with $d^{\prime}$ values significantly greater $(p<.05)$ than zero were 11,4 , and 0 for changes in pitch, roll, and translation, respectively. In contrast, the numbers of passive observers with significant $d^{\prime}$ values were 6,3 , and 0 for changes in pitch, roll, and translation, respectively.

Response bias. A four-factor (type of observation $x$ blackout duration $\times$ type of change $X$ consistency of change) ANOVA was conducted using the response bias measure $\beta$. The two-way interaction between consistency and type of change was significant $[F(2,22)=$ $9.54, p<.01]$. An analysis of the simple effects of consistency indicated a significant difference between inconsistent $(0.52)$ and consistent $(0.80)$ change $[F(1,11)=$ $11.4, p<.01]$. There were no other significant main effects or interactions.

The present results provide several important findings. First, active observation, relative to passive observation, resulted in greater sensitivity in detecting that a change had occurred. This conclusion is supported by the main effect of type of observation, the two-way interaction depicted in Figure 6, and the three-way interactions shown in Figures 5 and 8.

An interesting pattern of results occurred for blackout duration. Sensitivity decreased with an increase in blackout duration-a finding consistent with the results obtained in previous research (Lee, 1976; Schiff \& Detweiler, 1979; Thomson, 1983). The present results also suggest that a representational momentum effect occurred. Although the main effect of consistency was not significant, significantly greater sensitivity in detecting inconsistent change, relative to consistent change, was found in the two-way interactions illustrated in Figures 4 and 7 and in the three-way interactions depicted in Figures 5 and 8 . The present results also suggest that a representational momentum effect occurred only for short blackout durations (see Figure 4).

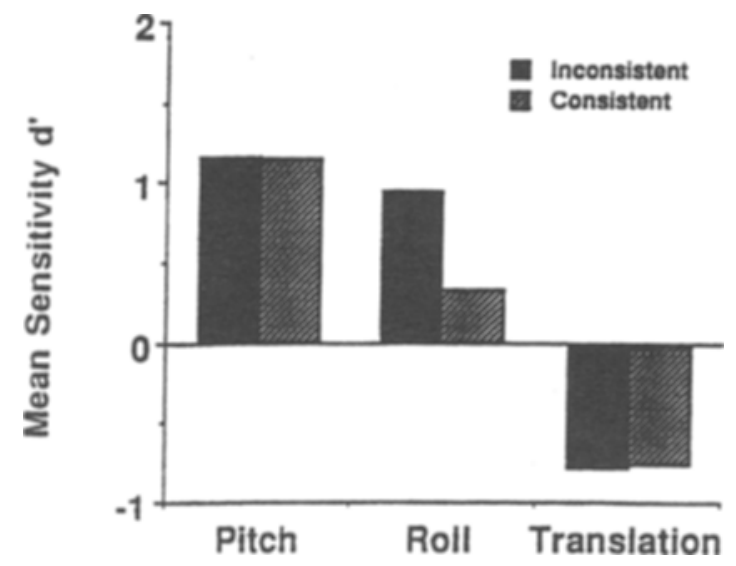

Figure 7. Effects of consistency of change and type of change on sensitivity in Experiment 1.
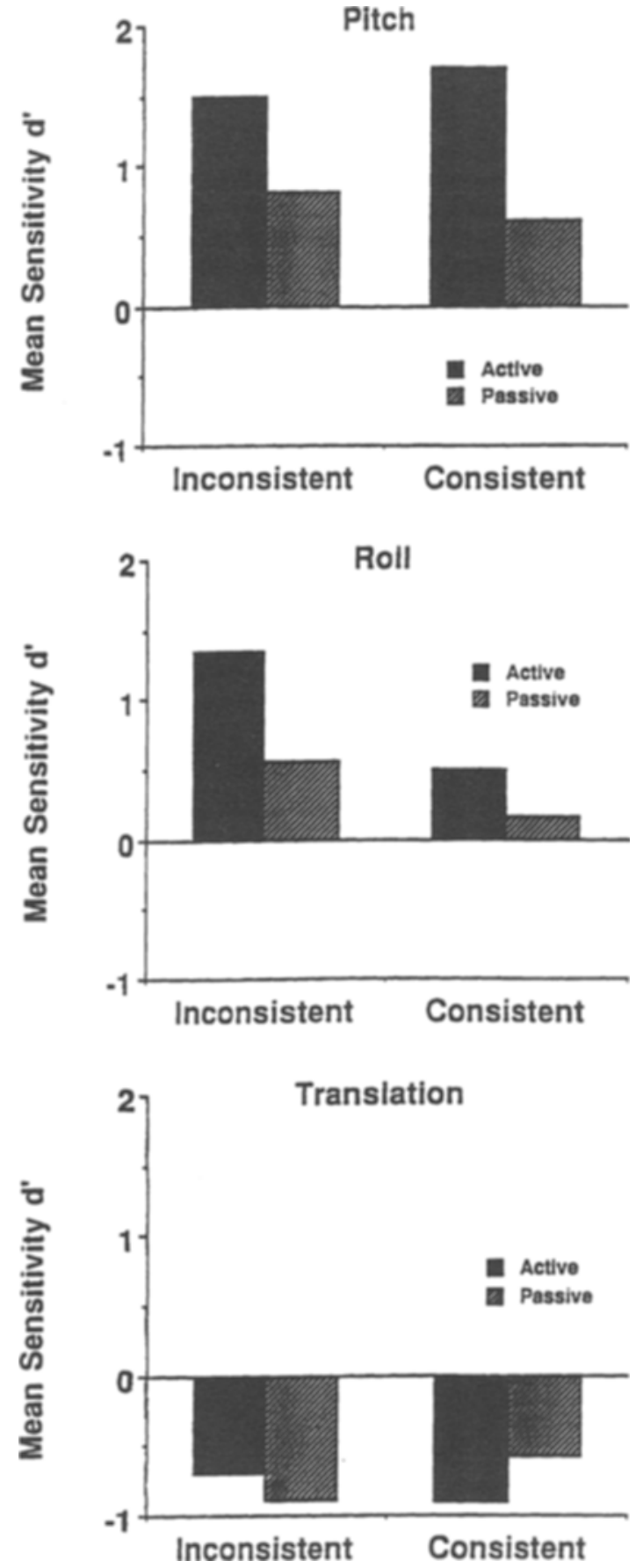

Figure 8. Effects of type of observation, consistency of change, and type of change on sensitivity in Experiment 1.

Finally, sensitivity varied as a function of type of change (roll, pitch, or translation). It should be mentioned, however, that no attempt was made to precisely equate detectability of these changes when generating the display conditions. Thus, the effects of type of change might have been due to differences in the ability of observers to detect the different types of change across variations in blackout duration. 


\section{EXPERIMENT 2}

In Experiment 1, active observers performed a compensatory tracking task by adjusting the simulated orientation of the observer in response to perturbations produced by sum of sines functions. Previous research (Poulton, 1974) has suggested that compensatory tracking, the task in Experiment 1, involves different control strategies than does pursuit tracking - a task in which the subject is required to follow a moving target. In Experiment 2 , we examined the effects of active and passive observation in a pursuit task. The task of active subjects was to follow a moving target while attending to orientation. In order to motivate tracking performance, subjects were instructed to press a button when the target was positioned in the crosshairs on the display. Passive observers were instructed to view the display and attend to their orientation, and to press a button when the target was positioned in the crosshairs of the display.

Each trial followed the same sequence of events as in Experiment 1. Following a period of tracking, the screen was blacked out for a brief period and was replaced by a static image. The subjects were asked to indicate whether they were at the correct orientation. The same variables examined in Experiment 1 were examined in Experiment 2.

\section{Method}

Subjects. Thirty-five paid volunteers, who were undergraduate and graduate students at the University of Illinois, participated in this experiment. The subjects had normal or corrected-to-normal vision and were naive with regard to the purpose of the experiment. Data from 3 subjects were lost due to a computer error.

Design. The design of this experiment was identical to that of Experiment 1. Five independent variables were examined: change (present or absent), type of observation (active control or passive observation), blackout duration $(1,2$, or $4 \mathrm{sec}$ ), type of change (rotation about the horizontal axis [pitch], rotation about the depth axis [roll], or translation in depth), and consistency of change with prior motion (consistent or inconsistent). Type of observation was manipulated between subjects. Presence or absence of change, blackout duration, type of change, and consistency of change were varied within subjects.

Stimuli. The stimuli were identical to the displays used in Experiment 1 with the following exceptions. The simulated position of the observer was not perturbed by simulated wind gusts. Instead, a target (a representation of a twin-engine aircraft) was present in the display. The motion of the target was determined by five-wave sum-of-sines forcing functions (see Table 1) that were used as input to the flight equations for the target aircraft. Shots fired by the subject were represented by moving red dots. If the shot "hit" the other aircraft, a fireball was drawn for $500 \mathrm{msec}$. Shots lasted $5 \mathrm{sec}$ or until the explosion (following a hit) ended. In addition, the position of the observer and the target were constrained to a separation of between 305 and $762 \mathrm{~m}$. Finally, maximum pitch and roll for both the target and the observer were limited to between $-30^{\circ}$ and $+30^{\circ}$.

Apparatus. The apparatus used in Experiment 2 was identical to that used in Experiment 1.

Procedure. Prior to the experiment, the subjects were told they were to be either pilots (active controllers) or copilots (passive observers). All subjects read the same set of instructions so that all observers would understand both their own tasks as well those of the other observers. The procedure was identical to that of Experiment 1 with the following exceptions. The subjects were told that their tasks were to shoot the target aircraft as often as possible and to attend to their orientation in space. They were also instructed that, at some point in each trial, a blackout would take place and that they would be required to detect and correct any unexpected changes that occurred during the blackout. They were challenged to perform as well as possible on both the shooting task and the orientation task. Active subjects were instructed to track the target aircraft so as to keep it lined up in their sights (the crosshair in the center of the display) for as much of the trial as possible. The subjects were also told that the target aircraft would move erratically to avoid them. As in Experiment 1, following each blackout, the subject was required to indicate with a buttonpress whether their aircraft was in the correct orientation. In addition, active observers were instructed not to initiate control responses during the blackout.

In this experiment, each subject first viewed a block of 30 practice trials. The greater number of practice trials used in the present experiment, relative to the first experiment, was intended to ensure that the subjects had sufficient practice with the difficult control demands of this experiment. The subjects then performed four additional blocks of 30 trials, two blocks on the first day and two more on a second day. Following each block, the subjects were given feedback on average performance on the shooting task, relative to other subjects in their viewing condition. No performance feedback was given for the orientation task.

The control responses of the subjects were monitored by the experimenter in the same way as they were in Experiment 1 . As in Experiment 1, the subjects who initiated control changes during the blackout were not included in the study. All subjects complied with the instructions, and, thus, no data were discarded as a result of inappropriate control changes.

\section{Results and Discussion}

As in Experiment 1, sensitivity $\left(d^{\prime}\right)$ in detecting that a change had occurred and response bias $(\beta)$ were calculated for each subject in Experiment 2 and examined in a four-factor (type of observation $\times$ blackout duration $\times$ type of change $x$ consistency of change) ANOVA. In addition, type of observation was analyzed as a repeated measures variable.

Sensitivity. A significant main effect for type of observation was found $[F(1,15)=11.08, p<.01]$, with greater sensitivity for active observers $(0.53)$ than for passive observers $(-0.03)$. The main effect of blackout duration was significant $[F(2,30)=4.61, p<.05]$. Post hoc comparisons indicated significant differences $(p<$ $.05)$ between the $1-\sec (0.43)$ and the 4-sec $(0.02)$ blackout duration. The main effect of consistency was significant $[F(1,15)=5.82, p<.05]$, with greater sensitivity in detecting inconsistent change (0.35) than in detecting consistent change (0.13). Finally, a significant main effect for type of change occurred $[F(2,30)=55.29, p<$ $.001]$. Post hoc comparisons indicated significant $(p<$ .05 ) differences in sensitivity between all pairwise comparisons of pitch $(1.0)$, roll $(-0.45)$, and translation (0.17).

The two-way interaction between type of observation and blackout duration was significant $[F(2,30)=4.61$, $p<.05]$ (see Figure 9). An analysis of the simple effects 
of type of observation, for different blackout durations, revealed significantly greater sensitivity for active observers $(0.8)$ than for passive observers $(0.06)$ at the 1 -sec blackout duration $[F(1,15)=11.9, p<.01]$ and greater sensitivity for active observers $(0.38)$ than for passive observers $(-0.1)$ at the 4 -sec blackout duration $[F(1,15)=14.3, p<.01]$.

The two-way interaction between type of observation and type of change was also significant $[F(2,30)=7.73$, $p<.01$ ] (see Figure 10). An analysis of the simple effects of type of observation indicated significantly greater sensitivity for active observers $(0.67)$ than for passive observers $(-0.31)$ for changes in translation $[F(1,15)=6.9, p<.01]$ and greater sensitivity for active observers (1.49) than for passive observers $(0.52)$ for changes in pitch $[F(1,15)=16.8, p<.01]$.

A significant two-way interaction was found between the type of change and blackout duration $[F(4,60)=$ $4.78, p<.01$ ] (see Figure 11). An analysis of the simple effect of blackout duration for changes in translation was significant $[F(2,30)=6.59, p<.025]$, with a significant difference $(p<.05)$ in sensitivity between the $1-\sec (0.56)$ and the 4-sec $(-0.26)$ blackout duration. An analysis of the simple effect of blackout duration for changes in pitch was also significant $[F(2,30)=5.74$, $p<.025$, with significant differences $(p<.05)$ in sensitivity between the $1-\sec (1.27)$ and the $4-\sec (0.63)$ blackout duration and between the 2-sec (1.11) and the $4-\sec (0.63)$ blackout duration.

Finally, the two-way interaction between consistency and type of change was significant $[F(2,30)=5.86, p<$ .025] (see Figure 12). An analysis of the simple effects of consistency indicated significantly greater sensitivity for inconsistent changes $(0.46)$ than for inconsistent changes $(-0.1)$ in translation $[F(1,15)=12.09, p<.01]$.

An analysis was also performed to determine whether the sensitivity of active and passive observers were significantly greater than zero (Marascuillo, 1970) for the three different types of change (roll, pitch, and transla-

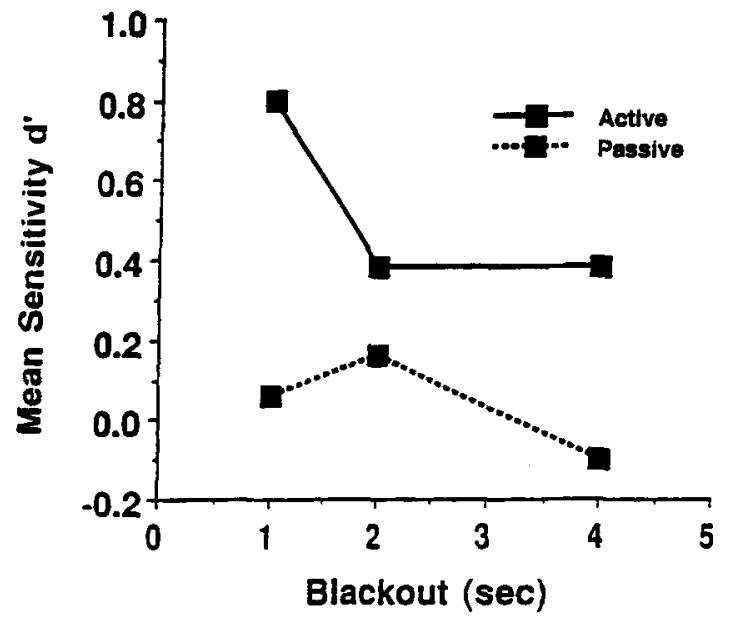

Figure 9. Effects of type of observation and blackout duration on sensitivity in Experiment 2.

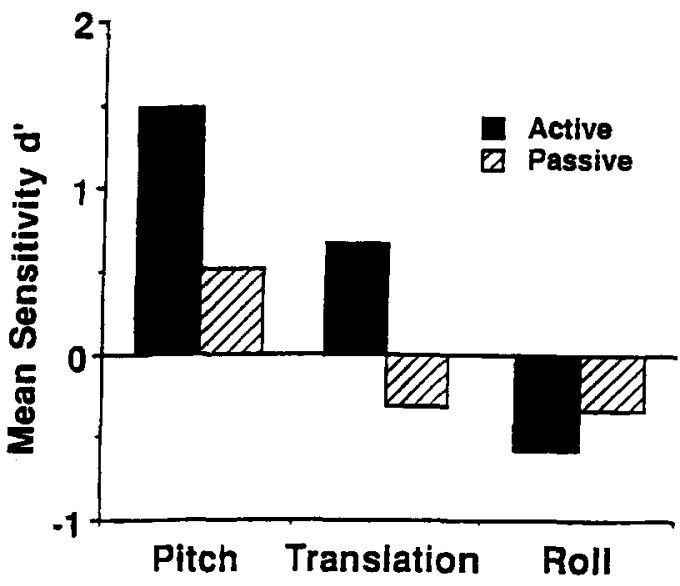

Figure 10. Effects of type of observation and type of change on sensitivity in Experiment 2.

tion). For active observers, the numbers of subjects with $d^{\prime}$ values significantly greater $(p<.05)$ than zero were 12,4 , and 0 for changes in pitch, translation, and roll, respectively. In contrast, the numbers of passive observers with significant $d^{\prime}$ values were 3,1 , and 0 for changes in pitch, translation, and roll, respectively.

Response bias. A four-factor (type of observation $x$ blackout duration $X$ type of change $X$ consistency of change) ANOVA was conducted using the response bias measure $\beta$. The main effect of consistency was significant $[F(1,15)=5.89, p<.05]$. Mean response bias for inconsistent and consistent change was .73 and .91, respectively. In addition, the two-way interaction between consistency and blackout duration was significant $[F(2,30)=3.99, p<.05]$. An analysis of the simple effects of consistency indicated a significant difference between inconsistent $(0.73)$ and consistent $(0.99)$ change for the 2-sec blackout duration $[F(1,15)=5.16$, $p<.05]$ and between inconsistent $(0.64)$ and consistent (1.08) change for the 4-sec blackout duration $[F(1,15)=$ $6.59, p<.025]$. There were no other significant main effects or interactions.

Overall, the results suggest greater sensitivity for active observation than for passive observation. This conclusion is supported by the significant main effect of the type of observation and the interaction between the type of observation and the type of change depicted in Figure 10. According to this interaction, increased sensitivity for active observation occurred for changes in translation and pitch.

An alternative explanation for the superior sensitivity of active observers in Experiment 2 concerns the requirement that the subjects detect changes in orientation and respond when the target was centered in the display. It is possible that performance differences between active and passive observers for the orientation task might have been due to different strategies employed to perform both tasks. For example, passive observers might have adopted a strategy in which they attended primarily to the target task rather than one in which they at- 


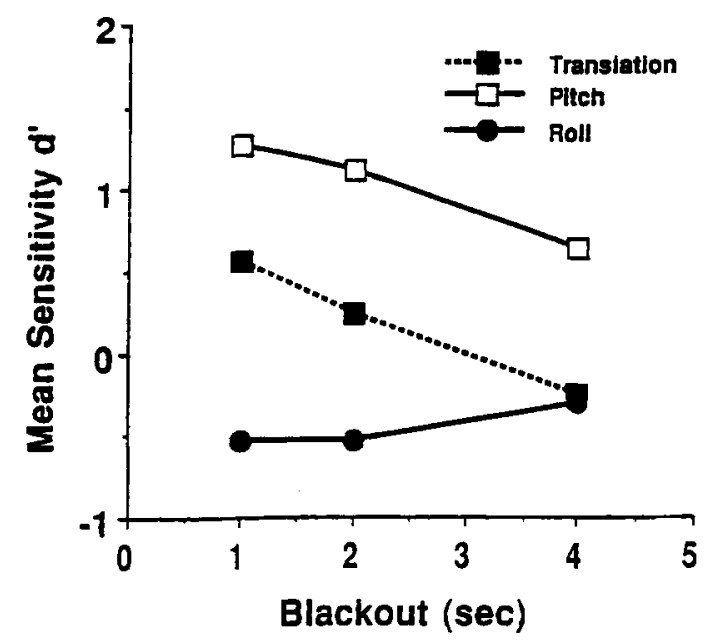

Figure 11. Effects of type of change and blackout duration on sensitivity in Experiment 2.

tended to both orientation and target tasks. If such a strategy were employed by passive observers, then performance in detecting orientation changes may have decreased at the cost of increased performance in responding to the target. Thus, the use of different strategies by active and passive observers in performing both tasks could account for the different orientation task results for these two groups. To examine this issue, we conducted a $t$ test to determine whether performance differed for active and passive observers in responding to the target. The $t$ test was not significant $[t(15)=-1.64, p<.12]$, suggesting that it is unlikely that the differences between active and passive observers in the orientation task were a result of a performance tradeoff in performing both tasks.

The results of Experiment 2 also provide evidence of a representational momentum effect. This conclusion is supported by the significant main effect of the consistency of change and the two-way interaction between the consistency of change and the type of change. Ac-

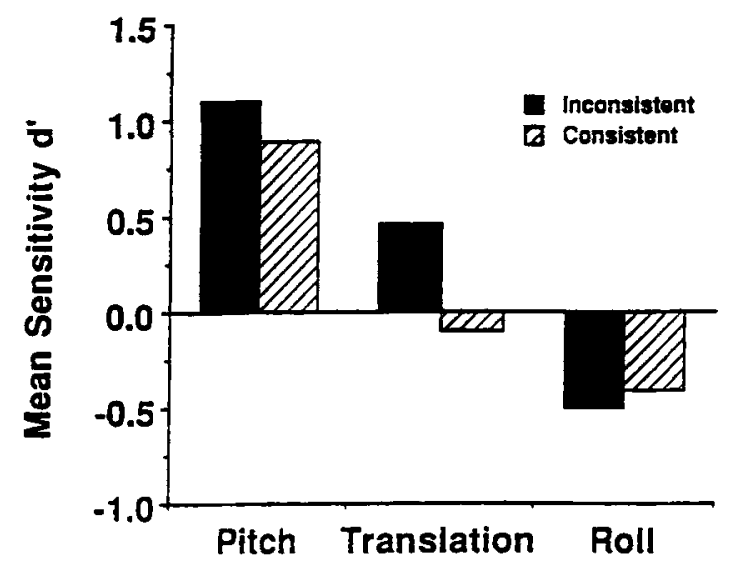

Figure 12. Effects of consistency of change and type of change on sensitivity in Experiment 2. cording to this interaction, a representational momentum effect was found only for changes in translation.

Control study. Although active observers were explicitly instructed not to initiate control changes during the blackout period, it is possible that control changes may have been detected during this period. This could have given active observers an advantage since the passive observers would lack information about these control changes. To ensure that the difference in performance between active and passive observers was not attributable to control changes during the blackout period, we replicated selected conditions from Experiment 2 with the following modification: Stick control was not sampled during the blackout and, thus, had no effect on the position or orientation of the simulation.

The subjects were 8 undergraduates at the University of Illinois who were paid for their participation. All of the subjects were naive with regard to the purposes of the experiment and had normal or corrected-to-normal vision. None of the subjects had participated in Experiment 1 or 2 . The control study examined detecting changes in pitch for the 1- and 2-sec blackout durations. These conditions were chosen because they produced the largest performance differences between passive and active subjects in Experiment 2. Four independent variables were examined: type of observation (active vs. passive), presence or absence of change, blackout duration ( 1 or $2 \mathrm{sec}$ ), and consistency of change (inconsistent or consistent). The task of the subject was identical to the task used in Experiment 2: The subjects were required to press a button when a target was aligned with the center of the display and to detect any changes in orientation following the blackout. The procedure was the same as that in Experiment 2 with the following exceptions: During the blackout period, stick control was not measured. The subjects were instructed that only a change in pitch could occur. In addition, the subjects received 20 practice trials and 8 replications for each combination of blackout duration, presence of change, and consistency of change.

Overall, active observers had greater sensitivity at detecting a change than did passive observers $[F(1,3)=$ $17.59, p<.025]$. Average $d^{\prime}$ values for active and passive observers were 2.22 and 1.08, respectively. All 4 of the active observers had $d^{\prime}$ values significantly greater $(p<.05)$ than zero, whereas 3 of the 4 passive observers had $d^{\prime}$ values significantly greater than zero. No significant difference $(p<.05)$ was found between active and passive observers for the secondary task. These results indicate that the greater sensitivity of active observers in detecting a change in orientation cannot be due to the introduction of control changes by the active observers during the blackout period.

\section{GENERAL DISCUSSION}

The present results indicate that active observation, relative to passive observation, is beneficial in detecting a change in extrapolated orientation. In Experiment 1, 
active observers were more sensitive than passive observers in detecting that a change had occurred. In addition, active observers, relative to passive observers, had greater sensitivity in detecting a change for both inconsistent and consistent trials across variations in blackout duration (see Figure 5). Active observers also demonstrated greater sensitivity in detecting inconsistent changes in pitch and roll and consistent changes in pitch than did passive observers (see Figure 8). In Experiment 2 , active observers had greater sensitivity, across all conditions examined, than did passive observers. Significantly greater sensitivity for active observers was found for the 1- and 4-sec blackout durations (see Figure 9) and for detecting changes in translation and pitch (see Figure 10). Results indicating significantly greater sensitivity for passive observers, relative to active observers, did not occur in any of the comparisons examined in Experiment 1 or 2 . These findings, taken together, indicate the benefit of active observation and support the proposal by Gibson (1966, 1979) of the importance of active observation.

A second important conclusion is that sensitivity in detecting a change decreased with an increase in the duration of blackout. This conclusion is supported by the significant main effect of blackout found in both experiments - the significant decrease in sensitivity with an increase in blackout duration, both for inconsistent and consistent change (illustrated in Figures 4 and 5) and for changes in translation or pitch (see Figure 11). In addition, a significant decrease in overall sensitivity occurred only for active observers in Experiment 2 (see Figure 9). These results are consistent with the results of previous studies (Lee, 1976; Schiff \& Detweiler, 1979; Thomson, 1983) and demonstrate that the time course of extrapolating orientation is relatively short.

A third important conclusion is that the observers demonstrated a representational momentum effect for extrapolating orientation following a blackout. In Experiment 1 , the subjects were significantly more sensitive at detecting inconsistent changes than they were at detecting consistent changes at the 1-sec blackout duration (see Figures 4 and 5) and at detecting inconsistent changes in roll (see Figure 7). In Experiment 2, the subjects were more accurate at detecting inconsistent changes than they were at detecting consistent changes, especially if the change involved translation (see Figure 7). These results, taken together, indicate that a representational momentum effect occurred for extrapolated orientation and suggest that the dynamics specified by optical flow are incorporated in the extrapolation of orientation in the immediate future.

Finally, a different pattern of results occurred in both experiments for the type of change. In Experiment 1, sensitivity was significantly worse for detecting changes in translation. However, in Experiment 2, sensitivity was significantly worse for detecting changes in roll. One explanation that may account for the different results in Experiments 1 and 2 concerns the type of tracking task required in each experiment. In Experiment 1, the task of the active observer was to maintain a heading direction. This task required responding to changes in pitch and roll. Translation was not relevant to the task of compensatory tracking. In contrast, pitch and translation (distance from the target) were critical to the task of tracking and shooting the target examined in Experiment 2. Roll information was not directly relevant to these tasks. This suggests that the differences in detecting a change, as a function of the type of change, may be due, in part, to the type of information critical to the task performed by an active observer. An important issue for future research would be to investigate this issue in greater detail.

In summary, the present results indicate that active observation, relative to passive observation, can result in improved performance in detecting changes in orientation. Our results are consistent with the proposal that active observers are more accurate at extrapolating orientation than are passive observers. This provides support for the proposal by Gibson $(1966,1979)$ that active control, relative to passive observation, can result in improved performance in perceptual tasks. In addition, the present findings provide support for the proposal that information specifying the dynamics of active control may be incorporated in extrapolating orientation.

\section{REFERENCES}

Armstrong, H. G. (1939). Principles and practice of aviation medicine. Baltimore, MD: Williams \& Wilkins.

Brooks, R. A. (1991). New approaches to robotics. Science, 253, 1227-1232.

Dhenin, G. (Ed.). (1978). Aviation medicine: Physiology and human factors. London: Tri-Med Books.

FLACH, J. M., \& WARREN, R. (1993). Active psychophysics: The relation between mind and what matters. In J. M. Flach, P. A. Hancock, J. K. Caird, \& K. J. Vicente (Eds.), An ecological approach to human machine systems I: Global perspectives (pp. 118-132). Hillsdale, NJ; Erlbaum.

FREYD, J. J. (1987). Dynamic visual representation. Psychological Review, 94, 427-438.

FREYD, J. J., \& FINKE, R. A. (1984). Representational momentum. Journal of Experimental Psychology: Learning, Memory, \& Cognition, 10, 126-132.

FREYD, J. J., \& FinKE, R. A. (1985). A velocity effect for representational momentum. Bulletin of the Psychonomic Society, 23, 443-446.

FREYD, J. J., \& Johnson, J. Q. (1987). Probing the time course of representational momentum. Journal of Experimental Psychology: Learning, Memory, \& Cognition, 13, 259-268.

GiBson, J. J. (1962). Observations on active touch. Psychological Review, 69, 477-491.

GiBson, J. J. (1966). The senses considered as perceptual systems. Boston, MA: Houghton Mifflin.

GrBson, J. J. (1979). The ecological approach to visual perception. Boston, MA: Houghton Mifflin.

JAGACINSKI, R. J. (1977). A qualitative look at feedback control as a style of describing behavior. Human Factors, 19, 33 1-347.

JAGACINSKI, R. J., JOHNSON, W. W., \& Miller, R. A. (1983). Quantifying the cognitive trajectories of extrapolated movements. Journal of Experimental Psychology: Human Perception \& Performance, 9 , 43-57.

KELLY, M. H., \& FREYD, J. J. (1987). Explorations of representational momentum. Cognitive Psychology, 19, 369-401.

KePPEL, G. (1991). Design and analysis. Englewood Cliffs, NJ: Prentice Hall.

LEE, D. N. (1976). A theory of visual control of braking based on information about time-to-collision. Perception, 5, 437-459. 
Marasculllo, L. A. (1970). Extensions of the significance test for oneparameter signal detection hypotheses. Psychometrika, 35, 237-243.

MCRuer, D. (1980). Human dynamics in man-machine systems. Automatica, 16, 237-253.

OMAN, C. M. (1982). A heuristic mathematical model for the dynamics of sensory conflict and motion sickness. Acta Oto-Laryngologica (Suppl. 392).

Poulton, E. C. (1974). Tracking skill and manual control. New York: Academic Press.

REASON, J., \& Brand, J. J. (1975). Motion sickness. London: Academic Press.

RoLNICK, A., \& LUBOW, R. E. (1991). Why is the driver rarely motion sick? The role of controllability in motion sickness. Ergonomics, 34, 867-879.

RUNESON, S. (1975). Visual prediction of collision with natural and nonnatural motion functions. Perception \& Psychophysics, 18, 261-268.

SCHIFF, W., \& DetweIleR, M. L. (1979). Information used in judging impending collision. Perception, 8, 647-658.

STAPPERs, P. J. (1989). Forms can be recognized from dynamic occlusion alone. Perceptual \& Motor Skills, 68, 243-251.

Thомson, J. A. (1983). Is continuous visual monitoring necessary in visually guided locomotion? Journal of Experimental Psychology: Human Perception \& Performance, 9, 427-443.
TOATES, F. M. (1975). Control theory in biology and experimental psychology. London: Hutchinson Educational.

WARREN, R. (1988). Active psychophysics: Theory and practice. In H. K. Ross (Ed.), Fechner Day '88, Proceedings of the 4th Annual Meeting of the International Society for Psychophysics (pp. 47-52). Stirling, Scotland: University of Stirling.

WARREN, R., \& MACMILLAN, G. R. (1984). Altitude control using actiondemanding interactive displays: Toward an active psychophysics. Proceedings of the 1984 IMAGE3 Conference (pp. 37-51). Phoenix, AZ: Air Force Human Resources Lab.

Whitemead, S. D., \& Ballard, D. H. (1991). Learning to perceive and act by trial and error. Machine Learning, 7, 45-83.

\section{NOTE}

1. The present methodology ensured that the optic flow fields were identical for matched active and passive observers. However, the retinal flow fields for matched active and passive observers would be quite different.

(Manuscript received February 23, 1993; revision accepted for publication September 26, 1994.) 